\title{
DNA Fingerprinting Construction and Genetic Diversity Analysis for Azuki Bean (Vigna angularis) and Mung Bean (Vigna radiata L.) Germplasm Resources
}

\author{
Qiuzhu Zhao', Zhe Chen ${ }^{1}$, Mohamed A.S. Khalifa ${ }^{1}$, \\ Fengxiang Yin², Xiangchun $\mathrm{Qu}^{3}$, Jun Zhang ${ }^{4}$, Dan Yao ${ }^{1}$
}

10.18805/LRF-652

\begin{abstract}
Background: As a coarse grain variety, azuki beans and mung beans were widely grow in Asia. Due to the lack of management in the long-term cultivation process, leading to the mixed phenomenon among azuki bean and mung bean varieties, which seriously affects the cultivation and breeding of azuki beans and mung beans. The current study aims to construct the genetic map using SSR markers for the genetic classification of beans.

Methods: In 2019, 46 test materials were raised to extract the young leaves. The DNA fingerprinting of 46 cultivated azuki bean and mung bean resources were constructed using 38 pairs of SSR primers and their genetic diversity were analyzed. Azuki bean and mung bean varieties are divided by hierarchical clustering analysis.

Result: Totally 18 primers have shown polymorphism among the 38 primers and 188 polymorphic bands were amplified with average $\mathrm{PIC}$ of value 0.8302 . According to the $\mathrm{K}$ mean cluster analysis method, 23 azuki beans and 23 mung beans resources were grouped into three categories. According to the hierarchical cluster analysis method, azuki beans were divided into four categories and mung beans were divided into six categories. The 46 azuki bean germplasm resources could be distinguished by screening nine pairs of digital fingerprint constructed by core primers.
\end{abstract}

Key words: Azuki bean, Construction of fingerprints, Genetic diversity, Mung bean, SSR marking.

\section{INTRODUCTION}

Azuki beans and mung beans both medical and food plants, were grown mainly in China, South Korea, Japan, Nepal and Bhutan. From the azuki beans, China has abundant azuki bean and mung bean germplasm resources (Xu et al., 2008; Sato et al., 2016; Hou et al., 2019; Kita-Tomihara et al., 2019; Kim et al., 2020; Wu et al., 2021). According to statistics, there are 4053 Chinese azuki bean and mung bean germplasm resources collected in China representing $39 \%$ of the updated storage resources in the world, ranking the first (Wang et al., 2001). During long-term cultivation and breeding, the resources collection of wild bean lags behind and the cultivation of bean lacks proper management. This makes the development of Chinese azuki bean market slow. The mixed phenomenon between varieties is obvious, which hinders the cultivation of new varieties of azuki beans (Zhao et al., 2011).

The study of genetic diversity plays a major role in crop breeding. The most direct manifestation of genetic diversity is the genetic structure of the population, which has a great significance not only for germplasms resources evaluation and preservation, but also for their genes to converge into a treasure house of genetic diversity. The in-depth analysis of genetic diversity can be used as a tool and a goal of breeding programs in order to better serve crop breeding and breeding quality traits (Jacob et al., 2017). Genetic diversity studies using molecular markers can get rid of the environment, climate and other constraints as the extent of
${ }^{1}$ College of Life Sciences, Jilin Agricultural University, Changchun 130118, China.

2Baicheng Academy of Agricultural Sciences, 137000, China.

${ }^{3}$ Institute of Crop Breeding, Jilin Academy of Agricultural Sciences, 130119, China.

${ }^{4}$ College of Agronomy, Jilin Agricultural University, Jilin, Changchun 130118, China.

Corresponding Author: Dan Yao, College of Life Sciences, Jilin Agricultural University, Changchun 130118, China.

Email: dyao@jlau.edu.cn

How to cite this article: Zhao, Q., Chen, Z., Khalifa, M.A.S., Yin, F., Qu, X., Zhang, J. and Yao, D. (2022). DNA Fingerprinting Construction and Genetic Diversity Analysis for Azuki Bean (Vigna angularis) and Mung Bean (Vigna radiata L.) Germplasm Resources. Legume Research. 45(2): 135-142. DOI: 10.18805/LRF-652.

Submitted: 03-09-2021 Accepted: 18-11-2021 Online: 29-01-2022

genetic variation is revealed at the genomic level. Therefore, molecular markers technology has gradually become a reliable test method for germplasm resources determination and classification (Qiu et al., 1998).

Azuki beans and mung beans are affected by disease and environmental effects during theirs growth, too much water and heavy metals have their adverse effects on growth and physiology of plant (Saikia et al., 2021; Saleem et al., 2021). Mung bean V4785 has a dominant inherited disease resistance gene for powdery mildew. The genetic basis of 
powdery mildew resistance was studied and the four marker loci were obtained using the ISSR (inter-simple sequence repeat) and ISSR-RGA (ISSR-anchored resistance gene analog) markers (Tantasawat et al., 2021). Bean image in storage and rust, powdery mildew and brown spot in beans have been widely used and SSR markers have been widely used in genetic mapping and identifying species origin. Azuki bean SSR markers have been reported for the first time in 2004 and five azuki bean natural populations have been detected. A total of 49 pairs of polymorphic primers were screened from 50 pairs of SSR specific primers (Wang et al., 2004). Further changes were made and 205 azuki bean SSR markers were published on 11 chain groups (Luo et al., 2013). After the genetic map of Chinese cultivated beans was SSR labeled, the idea of separate division of azuki bean germplasm resources, have emerged based on genetic background and was put forward and widely recognized. Recent researchers began to attach importance to the genetic classification of azuki bean (Wang et al., 2009). Based on SSR marker analysis, it is concluded that cultivated beans originated mainly in China, while wild-type beans may originate in southwest China, Bhutan or southern Japan, which further unravels the mystery of the origin of azuki beans (Zhao et al., 2011). According to 217 pairs of primers (196 pairs of azuki bean primers and 21 pairs of mung bean primers), 145 bean resources were divided into wild type, semi-wild type, local variety and main variety and 77 pairs of SSR primers with polymorphism were selected from them ( 75 pairs of azuki bean primers and 2 pairs of mung bean primers) (Zeng et al., 2010). A total of 397 mung bean germplasm resources were SSR labeled by 58 pairs of polymorphic mung bean primers at home and abroad and 8 rare allelic variations were detected. The discovery of rare allelic variation provides a new research direction for plant growth and development (Qiao et al., 2015). Fingerprinting of 92 mung bean germplasm resources in Inner Mongolia was constructed using 100 pairs of mung bean SSR primers (Zhao et al., 2017). 40 pairs of primers with high polymorphism and clear bands amplified were screened, using a combination of magnetic bead enrichment and sequencing to analyze the genetic diversity of 90 mung bean resources from all over the country, a total of $3,275,355$ SSR sites were identified, from which 2,742 markers were designed and the primer polymorphism was as high as 57.33 (Ye et al., 2019).

Although some progress has been made in the study of azuki bean, it has been greatly affected by regional or genetic background. Especially, there is no related report on the fingerprint DNA of azuki bean germplasm resources with Jilin Province as the main research background. In this study, the genetic diversity of 46 azuki bean cultivars was analyzed and 38 pairs of SSR primers were used to construct their SSR digital fingerprints, which filled the gap of genetic background of azuki bean in Jilin Province, China. And provides theoretical basis for screening, utilization and genetic improvement of azuki bean germplasm resources.

\section{MATERIALS AND METHODS}

The 46 germplasms of azuki bean and mung bean (Table 1) were obtained from the Academy of Agricultural Sciences of Baicheng, Jilin Province. They were planted in Jilin Agricultural University teaching experimental $\left(43^{\circ} 88^{\prime} \mathrm{N}\right.$, $125^{\circ} 35^{\prime} \mathrm{E}$ ) base during May 15,2019 . The young leaves of the three-leaf stage were collected using tin foil paper, flash frozen in liquid nitrogen and stored at $-80^{\circ} \mathrm{C}$.

The leaf genome was extracted using Kangwei plant genome DNA extraction kit (Cat:C W0531M). Determination of DNA concentration and purity were estimated by using NanoDrop 2000C Thermo scientific analyzer and $1.0 \%$ agarose gel electrophoresis then stored at $-20^{\circ} \mathrm{C}$ for later use.

The reaction mixture $(20 \mu \mathrm{L})$ contained $10 \mu \mathrm{L}$ of Es Taq MasterMix, $0.4 \mu \mathrm{L}$ of forward primer $\left(20 \mathrm{ng} \cdot \mu \mathrm{L}^{-1}\right), 0.4 \mu \mathrm{L}$ of reverse primer $\left(20 \mathrm{ng} \cdot \mu^{-1}\right), 0.8 \mu \mathrm{L}$ of template DNA (10 $\left.\mathrm{ng} \cdot \mu \mathrm{L}^{-1}\right)$ and $8.4 \mu \mathrm{L}$ of $\mathrm{dd}_{2} \mathrm{O}$.

The amplification conditions included an initial denaturation step of $94^{\circ} \mathrm{C}$ for $5 \mathrm{~min}$, followed by 30 cycles of denaturation at $98^{\circ} \mathrm{C}$ for $10 \mathrm{~s}$ and annealing extension at $52 \sim 58^{\circ} \mathrm{C}$ for $30 \mathrm{~s}$, elongation at $72^{\circ} \mathrm{C}$ for $30 \mathrm{~s}$ and a final step at $72^{\circ} \mathrm{C}$ for $1 \mathrm{~min}$. PCR products at $94^{\circ} \mathrm{C}$ to degenerate

Table 1: The name of azuki bean and mung bean.

\begin{tabular}{|c|c|c|c|c|c|c|c|}
\hline \multicolumn{4}{|c|}{ Name of mung bean } & \multicolumn{4}{|c|}{ Name of azuki bean } \\
\hline 1 & Bai Lv 925 & 13 & Bai Lv 6 & 1 & Bai Hong 12 & 13 & Li Xiao Dou \\
\hline 2 & Bai Lv 985 & 14 & Tong Lv 918 & 2 & Bai Hong 11 & 14 & Pin Hong 2011-18 \\
\hline 3 & Bai Lv 522 & 15 & Ji Lv 03083 & 3 & Bai Hong 10 & 15 & Ji Hong 9218 \\
\hline 4 & Bai Lv 935 & 16 & Ji Lv 9 & 4 & Bai Hong 9 & 16 & Ji Hong 352 \\
\hline 5 & Bai Lv 15 & 17 & Ji Lv 8 & 5 & Bai Hong 8 & 17 & Ji Hong 0015 \\
\hline 6 & Bai Lv 14 & 18 & Ji Lv 7 & 6 & Bai Hong 7 & 18 & Zhong Hong 5 \\
\hline 7 & Bai Lv 13 & 19 & Liao Lv 8 & 7 & Bai Hong 6 & 19 & Liao Hong 08721 \\
\hline 8 & Bai Lv 12 & 20 & Liao Lv 3 & 8 & Bai Hong 5 & 20 & Ji Hong 10 \\
\hline 9 & Bai Lv 11 & 21 & Zhong Lv 11 & 9 & Bai Hong 4 & 21 & Ji Hong 8 \\
\hline 10 & Bai Lv 10 & 22 & Zhong Lv 8 & 10 & Bai Hong 3 & 22 & TangHong2010-23 \\
\hline 11 & Bai Lv 9 & 23 & Zhong Lv 5 & 11 & Bai Hong 2 & 23 & TangHong2010-12 \\
\hline 12 & Bai Lv 8 & & & 12 & Bai Xiao Dou & & \\
\hline
\end{tabular}


DNA Fingerprinting Construction and Genetic Diversity Analysis for Azuki Bean (Vigna angularis) and Mung Bean (Vigna...

for 5 min, then quickly cooled on ice and tested with a polyacrylamide gel. The silver staining method was used to display the color and the electrophoresis results were observed on a slide lamp (Gresshoff et al., 1991; Wang et al., 2017).

According to the polyacrylamide electrophoresis results, a band of the same fragment size was recorded as a marker allele and scored as 1 and 0 for a band, no band and a deletion. The direction of reading band was from top to bottom, the results were transformed into a string composed of 1 and 0 , the digital fingerprint of 46 varieties was formed. IBM SPSS Statistics V.23 software was used to carry out the $K$ value clustering analysis and the hierarchical clustering analysis on the genetic relationship between different varieties of beans based on pod length averages and pod number averages traits. Finally, the clustering map was constructed.

\section{RESULTS AND DISCUSSION}

\section{Detection of genome DNA quality of azuki and mung bean}

For qualitative detection of the genomic DNA of azuki and mung bean, $1 \%$ agarose gel electrophoresis was used. Part of the results are shown in Fig 1. Genome of azuki and mung bean leaves had good quality DNA with no dispersion. With regard to quantitative detection the A260/A280 ratio of genomic DNA between 1.78 and 1.91 , concentrations ranged from 15.9 to $199 \mathrm{ng} / \mu \mathrm{L}$, which meets the conditions of subsequent SSR-PCR gene amplification.

\section{SSR primer polymorphism analysis}

In total, 38 primer pairs were designed and screened: 12 pairs of azuki bean SSR primers and 26 pairs of mung bean SSR primers were screened in the laboratory. The results showed that the effective amplification rate of 12 pairs of azuki bean SSR primers was $100 \%$ and the polymorphism rate was $50 \%$, on the other hand the effective amplification rate of 26 pairs of mung bean SSR primers was $92.3 \%$ and the polymorphism rate was $46.2 \%$ (Fig 2).
A total of 18 primers (6 pairs of azuki bean SSR primers, 12 pairs of mung bean SSR primers) was polymorphic and stable SSR primers were selected for genetic diversity analysis of azuki bean germplasm. Use of the polymorphic markers resulted in $45.52 \%$ polymorphism rate, $3-25$ alleles in each primer, 11 Average polymorphism, 0.6157-0.9501 is the variation range of polymorphic information content (PIC) and 0.8302 is the average PIC value (Table 2).

\section{Cluster analysis of azuki and mung bean germplasm resources}

According to the $\mathrm{K}$ mean clustering method, the results show that the mung bean and azuki bean resources are both divided into three categories (Table 3). Mung beans are classified as follows: category I (Bai Lv 9, Liao Lv 3, Zhong Lv 11, Zhong Lv 8, Zhong Lv 5), category II (Bai Lv 985, Bai Lv 935, Bai Lv 925, Bai Lv 522, Bai Lv 15, Bai Lv 14, Bai Lv 8, Bai Lv 6, Ji Lv 9, Ji Lv 8, Ji Lv 7) and category III (Tong Lv 918, Bai Lv 13, Bai Lv 12, Bai Lv 11, Bai Lv 10, Ji Lv 03083 , Liao Lv 8). Azuki beans are classified as follows: category I (Bai Hong 12, Bai Hong 10, Bai Hong 8, Bai Hong 7, Bai Hong 4, Zhong Hong 5, Liao Hong 08721, Ji Hong 10, Ji Hong 8, Tang Hong 2010-23), category II (Bai Hong 11, Bai Hong 5, Bai Hong 3, Bai Xiaodou, Pin Hong 2011-18, Ji Hong 9218, Ji Hong 352, Ji Hong 0015) and category III (Bai Hong 9, Bai Hong 6, Bai Hong 2, Li Xiaodou, Tang Hong 2010-12). Hierarchical clustering analysis was using centroid clustering method to process data and map trees (Fig 3), euclidean distance (D) of standardized variables is used as a cluster statistics to classify the tested varieties, at $D=7.50$, the classification limits were intercepted and it can be seen from the cluster map that mung bean varieties can be divided into six categories: category I (Bai Lv 522, Bai Lv 6, Bai Lv 15, Ji Lv 7, Bai Lv 14, Bai Lv 935, Bai Lv 925, Bai Lv 985, Ji Lv 9, Ji Lv 8, Bai Lv 11, Bai Lv 10, Bai Lv 13, Bai Lv 12, Liao Lv 8), category II (Bai Lv 8, Tong Lv 918), category III (Bai Hong 6, Tang Hong 2010-12), category IV (Bai Hong 11, Ji Hong 9218), Category V (Bai Hong 9, Li Xiaodou, Bai Hong 2) and category VI (Bai Hong 3, Ji Hong 352, Bai Hong 5, Bai Xiaodou, Pin Hong 2011-18).

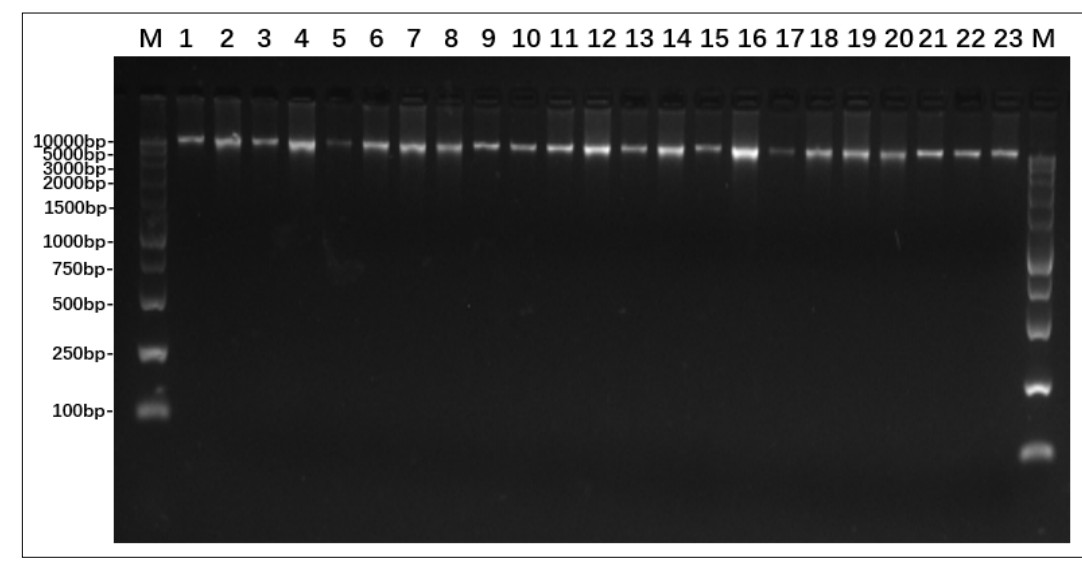

Fig 1: $2 \%$ agarose gel electrophoresis for detection of mung bean genome mass. Note: M: DL10K Plus DNA Marker; 1-23: Mung bean DNA. 


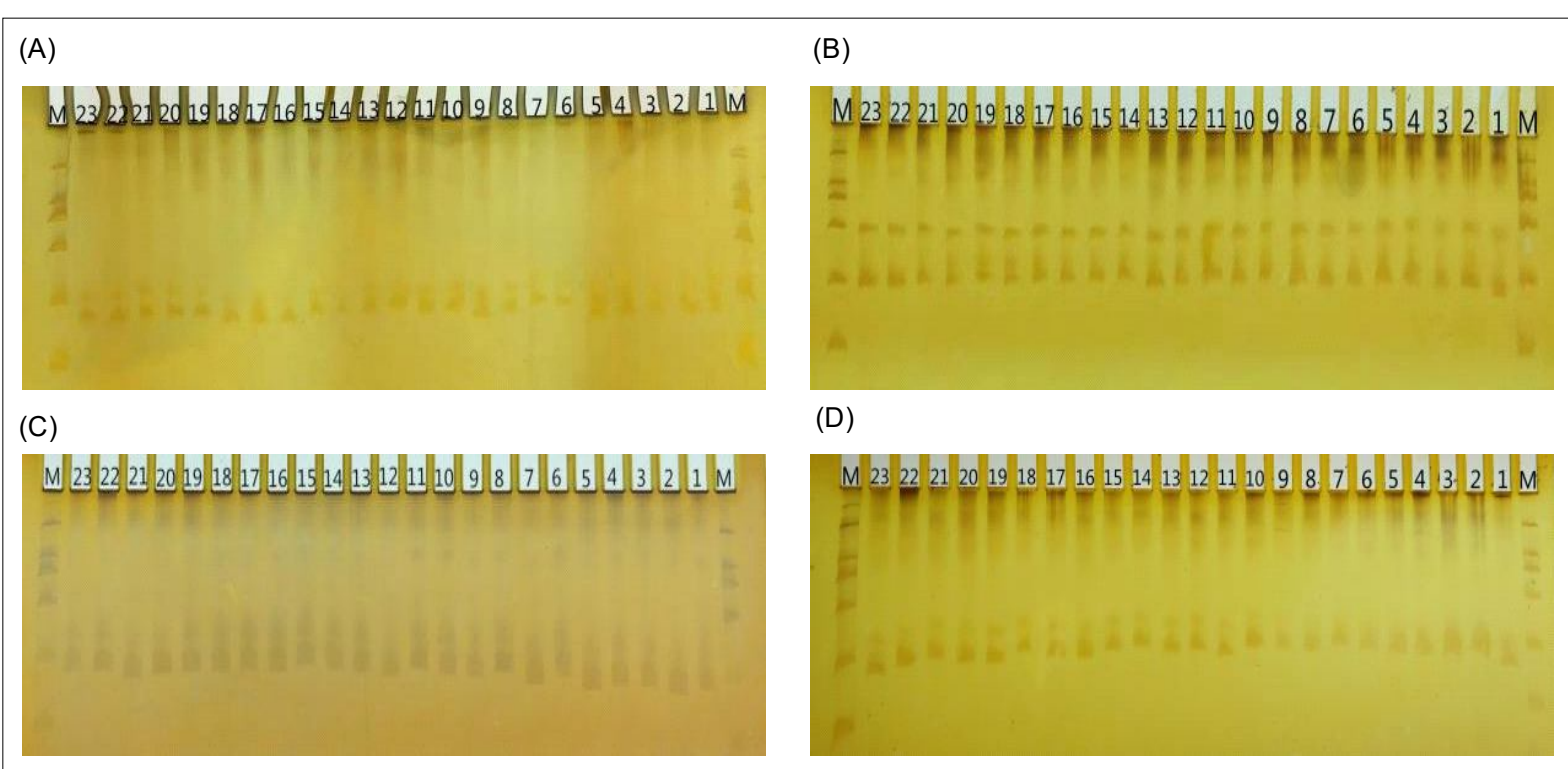

Fig 2: Partial SSR-PCR polyacrylamide gel electrophoresis of azuki bean and mung bean.

(A) Mung bean primer DMBSSR024. (B) Mung bean primer DMBSSR059.

(C) Azuki bean primer CEDG048. (D) Azuki bean primer CEDG151.

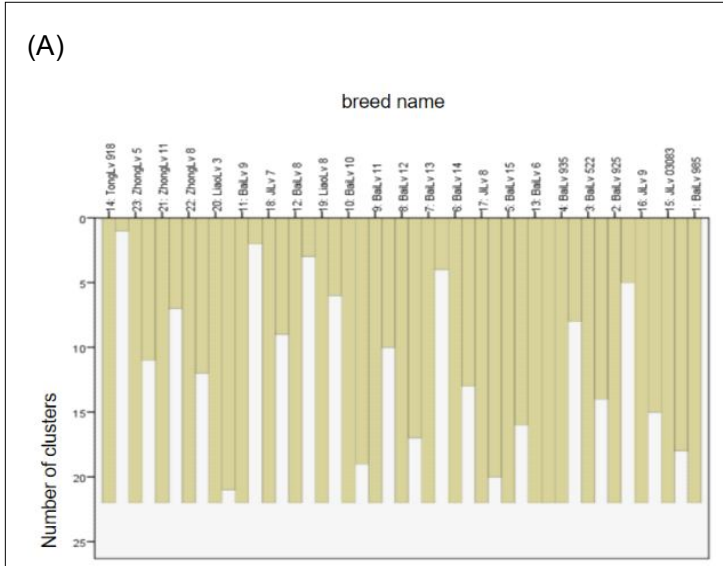

(C)

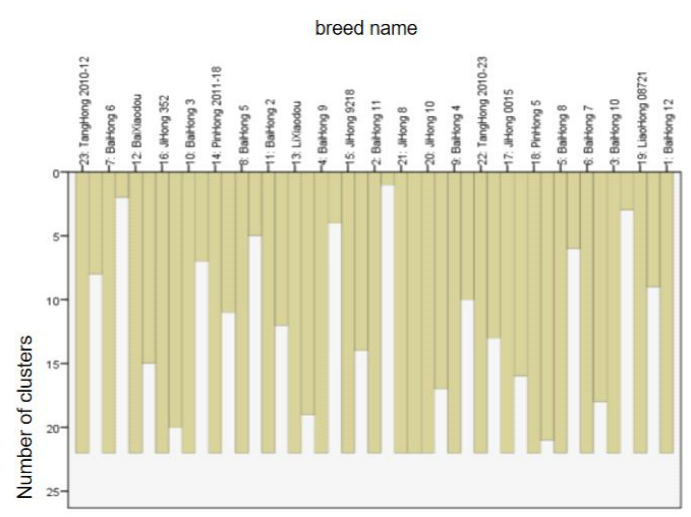

(B)

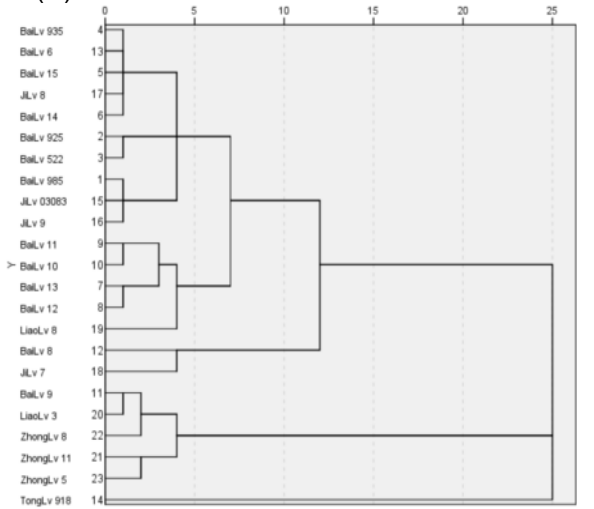

(D)

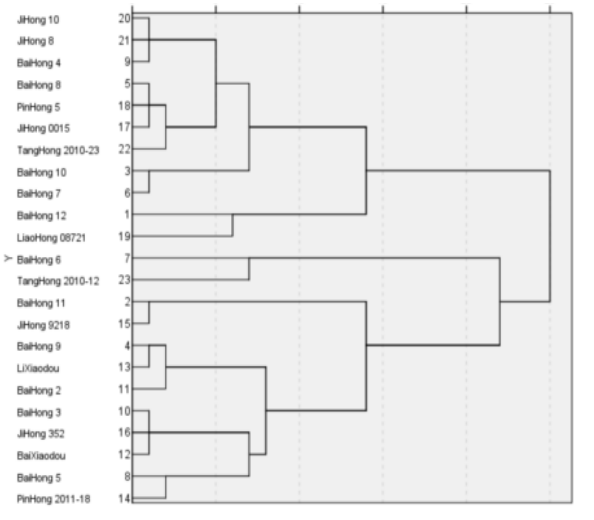

Bartong 50

Fig 3: Clustering analysis of mung beans and adzuki beans.

(A) Number of clustering clusters in mung beans. (B) Pedigree of mung beans using centroid junction.

(C) Number of clustering clusters in azuki beans. (D) Pedigree of azuki beans using centroid junction. 
DNA Fingerprinting Construction and Genetic Diversity Analysis for Azuki Bean (Vigna angularis) and Mung Bean (Vigna ...

Table 2: Characterization of 18 polymorphic primer.

\begin{tabular}{|c|c|c|c|c|c|}
\hline Primer & $\begin{array}{c}\text { Annealing } \\
\text { temperature } /{ }^{\circ} \mathrm{C}\end{array}$ & Equivalent & $\begin{array}{c}\text { Number of } \\
\text { polymorphic loci }\end{array}$ & $\begin{array}{l}\text { Polymorphism } \\
\text { rate } / \%\end{array}$ & $\begin{array}{c}\text { Polymorphism information } \\
\text { content/PIC }\end{array}$ \\
\hline CEDG133 & 54 & 44 & 15 & 34.1 & 0.9187 \\
\hline CEDG050 & 53 & 38 & 18 & 47.4 & 0.9215 \\
\hline CEDG020 & 53 & 36 & 16 & 44.4 & 0.8966 \\
\hline CEDG111 & 56 & 23 & 6 & 26.1 & 0.7612 \\
\hline CEDG151 & 52 & 23 & 8 & 34.8 & 0.8450 \\
\hline CEDG024 & 55 & 23 & 6 & 26.1 & 0.7146 \\
\hline DMBSSR001 & 52 & 23 & 3 & 13.0 & 0.6157 \\
\hline DMBSSR016 & 52 & 23 & 5 & 21.7 & 0.7070 \\
\hline DMBSSR024 & 55 & 23 & 7 & 30.4 & 0.7902 \\
\hline DMBSSR043 & 52 & 57 & 25 & 43.9 & 0.9501 \\
\hline MBSSR015 & 55 & 23 & 8 & 34.8 & 0.8015 \\
\hline MBSSR039 & 52 & 46 & 12 & 26.1 & 0.9433 \\
\hline MBSSR041 & 55 & 53 & 12 & 22.6 & 0.8622 \\
\hline MBSSR063 & 52 & 39 & 14 & 35.9 & 0.8994 \\
\hline MBSSR095 & 55 & 45 & 13 & 28.9 & 0.9017 \\
\hline MBSSR136 & 58 & 46 & 13 & 28.3 & 0.8866 \\
\hline MBSSR179 & 52 & 40 & 11 & 27.5 & 0.9013 \\
\hline MBSSR203 & 52 & 23 & 6 & 26.1 & 0.6276 \\
\hline Total & - & 628 & 198 & - & 14.9442 \\
\hline Average value & - & 35 & 11 & - & 0.8302 \\
\hline
\end{tabular}

Notes: Polymorphic information (polymorphism information content, PIC).

$\mathrm{PIC}=1-\sum(\mathrm{Pi}) 2$, (the ratio of the first allele to the total allele).

Table 3: K-means clustering analysis.

\begin{tabular}{|c|c|c|c|c|c|c|c|}
\hline Number & Name & Cluster & Distance & Number & Name & Cluster & Distance \\
\hline 1 & Bai Lv 985 & 2 & 1.267 & 24 & Bai Hong 12 & 1 & 1.295 \\
\hline 2 & Bai Lv 935 & 2 & 0.831 & 25 & Bai Hong 11 & 2 & 0.75 \\
\hline 3 & Bai Lv 925 & 2 & 0.763 & 26 & Bai Hong 10 & 1 & 0.709 \\
\hline 4 & Bai Lv 522 & 2 & 0.423 & 27 & Bai Hong 9 & 3 & 0.639 \\
\hline 5 & Bai Lv 15 & 2 & 0.253 & 28 & Bai Hong 8 & 1 & 0.958 \\
\hline 6 & Bai Lv 14 & 2 & 0.522 & 29 & Bai Hong 7 & 1 & 0.7 \\
\hline 7 & Bai Lv 13 & 3 & 0.972 & 30 & Bai Hong 6 & 3 & 1.451 \\
\hline 8 & Bai Lv 12 & 3 & 0.731 & 31 & Bai Hong 5 & 2 & 0.378 \\
\hline 9 & Bai Lv 11 & 3 & 0.620 & 32 & Bai Hong 4 & 1 & 0.32 \\
\hline 10 & Bai Lv 10 & 3 & 0.527 & 33 & Bai Hong 3 & 2 & 0.627 \\
\hline 11 & Bai Lv 9 & 1 & 0.439 & 34 & Bai Hong 2 & 3 & 0.863 \\
\hline 12 & Bai Lv 8 & 2 & 1.381 & 35 & Bai Xiaodou & 2 & 0.643 \\
\hline 13 & Bai Lv 6 & 2 & 0.348 & 36 & Li Xiaodou & 3 & 0.612 \\
\hline 14 & Ji Lv 03083 & 3 & 1.685 & 37 & Pin Hong 2011-18 & 2 & 0.666 \\
\hline 15 & Ji Lv 9 & 2 & 1.071 & 38 & Ji Hong 9218 & 2 & 1.021 \\
\hline 16 & Ji Lv 8 & 2 & 0.783 & 39 & Ji Hong 352 & 2 & 0.643 \\
\hline 17 & Ji Lv 7 & 2 & 0.269 & 40 & Ji Hong 0015 & 2 & 1.021 \\
\hline 18 & Tong Lv 918 & 2 & 1.640 & 41 & Zhong Hong 5 & 1 & 0.864 \\
\hline 19 & Liao Lv 8 & 3 & 0.504 & 42 & Liao Hong 08721 & 1 & 1.227 \\
\hline 20 & Liao Lv3 & 1 & 0.552 & 43 & Ji Hong 10 & 1 & 0.313 \\
\hline 21 & Zhong Lv 11 & 1 & 0.662 & 44 & Ji Hong 8 & 1 & 0.313 \\
\hline 22 & Zhong Lv 8 & 1 & 0.512 & 45 & Tang Hong $2010-23$ & 1 & 0.592 \\
\hline 23 & Zhong Lv 5 & 1 & 0.794 & 46 & Tang Hong 2010-12 & 3 & 0.705 \\
\hline
\end{tabular}


DNA Fingerprinting Construction and Genetic Diversity Analysis for Azuki Bean (Vigna angularis) and Mung Bean (Vigna...

\section{Map construction of DNA fingerprints of beans}

According to the allelic variation, PIC value and repeatability of the amplification of the 18 pairs of primers with best polymorphism will be selected. Nine pairs of core polymorphism primers (CEDG103, CEDG151, CEDG024, DMBSSR016, DMBSSR024, MBSSR015, MBSSR095, MBSSR136 and MBSSR179) were selected to construct the fingerprint of 46 main varieties of azuki bean and mung bean (Table 4). The difference between the fingerprints of 46 varieties indicates that the digital fingerprints of 46 varieties can be constructed by using these nine pairs of primers (Table 5 ).

In the process of species selection and evolution, organisms constantly accumulate accidental simple or complex mutations in which this mutation can be inherited to offspring which leads to different levels of genetic differences and the formation of abundant genetic diversity within or between species (Bai, 2014). Changes in plant phenotypes cannot fully respond to genetic variation so morphological level identification of biodiversity is limited. SSR molecular markers technology can directly detect the changes and differences in molecular structure at genetic level with high sensitivity and convenient operation overcoming the deficiency of the morphological strategies (Nordborg et al., 2000; Zhao et al., 2011). SSR primers were used to analyze the correlation between grain color and 100 grain weight traits of azuki bean varieties from different regions. The tested materials were divided into four groups deploying 73 SSR markers polymorphic sites amplification (Meng et al., 2008). By cluster analysis, some similar data

Table 4: 30 alleles of 9 SSR Loci.

\begin{tabular}{lccccc}
\hline Serial number & Allelic variation locus & Serial number & Allelic variation locus & Serial number & Allelic variation locus \\
\hline 1 & CEDG103-114 & 11 & MBSSR095-145 & 21 & MBSSR015-129 \\
2 & CEDG103-154 & 12 & MBSSR095-147 & 22 & MBSSR015-185 \\
3 & CEDG151-179 & 13 & MBSSR095-150 & 23 & MBSSR095-112 \\
4 & CEDG151-275 & 14 & MBSSR095-155 & 24 & MBSSR095-114 \\
5 & CEDG024-119 & 15 & MBSSR136-142 & 25 & MBSSR095-142 \\
6 & CEDG024-154 & 16 & DMBSSR024-177 & 26 & MBSSR136-194 \\
7 & CEDG024-159 & 17 & DMBSSR024-184 & 27 & MBSSR179-117 \\
8 & CEDG024-168 & 18 & DMBSSR024-222 & 28 & MBSSR179-177 \\
9 & DMBSSR016-149 & 19 & DMBSSR024-225 & 29 & MBSSR179-155 \\
10 & DMBSSR016-156 & 20 & DMBSSR024-235 & 30 & MBSSR179-157 \\
\hline
\end{tabular}

Table 5: 23 azuki bean DNA fingerprints.

\begin{tabular}{llll}
\hline Name & Digital number & Name & Digital number \\
\hline Bai Hong 12 & 101000000000000000100000000000 & Bai Lv 925 & 000000010010000000000010000100 \\
Bai Hong 11 & 000010000000000000000000000000 & Bai Lv 985 & 000000000001000000010000100000 \\
Bai Hong 10 & 000000000000000000000000000000 & Bai Lv 522 & 000000000000000100000001000010 \\
Bai Hong 9 & 000000000000000000000000000000 & Bai Lv 935 & 010001000000000000000000000000 \\
Bai Hong 8 & 000000000000000000000000000000 & Bai Lv 15 & 000000000000000000000010000000 \\
Bai Hong 7 & 000000000000000000000001000000 & Bai Lv 14 & 000000000000010000010000100000 \\
Bai Hong 6 & 000100000000000000000000000000 & Bai Lv 13 & 001000000000001000000100000000 \\
Bai Hong 5 & 000000000000000000000000000000 & Bai Lv 12 & 000000000000000100000000000001 \\
Bai Hong 4 & 000000000000000000000000000000 & Bai Lv 11 & 000000000100000000000000010000 \\
Bai Hong 3 & 000000000000000000000000000000 & Bai Lv 10 & 000000000010000000010000100100 \\
Bai Hong 2 & 000000001000000000010000100000 & Bai Lv 9 & 000000000001000010000001000010 \\
Bai Xiaodou & 010010000000000000000000000000 & Bai Lv 8 & 000100000100100000001000000000 \\
Li Xiaodou & 000000000000000000000000000000 & Bai Lv 6 & 010001000000100000010010100000 \\
Pin Hong 2011-18 & 000000010000000000000000000000 & Tong Lv 918 & 000000000100000000000001000010 \\
Ji Hong 9218 & 000000000000000000000000000000 & Ji Lv 03083 & 010001000000000000000000000000 \\
Ji Hong 352 & 000001100000000100000000000000 & Ji Lv 9 & 000000000000000000001100000000 \\
Ji Hong 0015 & 010010000000000000000000000000 & Ji Lv 8 & 000000000011000010010000100100 \\
Zhong Hong 5 & 000100000000000000000000000000 & Ji Lv 7 & 000100000000000000000000000000 \\
Liao Hong 08721 & 000000000000000000001100000000 & Liao Lv 8 & 000000000000000001001000000000 \\
Ji Hong 10 & 000000000000000000010000100000 & Liao Lv 3 & 000000000000000000000000000000 \\
Ji Hong 8 & 000000000000000000000000000000 & Zhong Lv 11 & 000000001000000000000000000000 \\
Tang Hong 2010-23 & 000000000000000100000000000000 & Zhong Lv 8 & 010001000100000000000000000000 \\
Tang Hong 2010-12 & 000000000000000000000000000000 & Zhong Lv 5 & 100000000100000000100001010010 \\
\hline
\end{tabular}


DNA Fingerprinting Construction and Genetic Diversity Analysis for Azuki Bean (Vigna angularis) and Mung Bean (Vigna...

members can be classified centrally to better understand the relationships between the species.

In our present investigation, 18 pairs of specific primers were selected and 628 alleles were amplified in azuki bean and mung bean. Each pair of primers detected 25-37 loci, with an average of 35 alleles and 0.8302 as PIC values, which was higher than that of previous studies (Qiao et al., 2020). The polymorphism ratios of azuki bean and mung bean primers were $50 \%$ and $46.2 \%$, respectively. High polymorphism, strong specificity and good reproducibility of primers were beneficial for variety identification. Therefore, they could be used as core primers for genetic analysis and fingerprinting of subsequent bean germplasms resources.

By means of $\mathrm{K}$ mean cluster analysis and stratified cluster analysis, the genetic relationship between different varieties of bean was further analyzed. Classification has some convergence in genetic types, the identification of materials from different regions at the same test site may have an impact on the morphological characteristics of certain bean plants. However, it is of biological significance to identify the phenotype and genetic stability of species in different environments (Bai et al., 2014). By means of $\mathrm{K}$ mean cluster analysis, they have similar morphological characteristics with bean varieties from the same origin. Based on hierarchical cluster analysis, the mung bean germplasm materials are divided into four groups, among which Ji Lv 03083 is a single group indicating that maybe evolve in different ecological environments or geographical isolation. Interestingly, Bai Hong 10, Bai Hong 12, Bai Hong 6, Bai Hong 11, Bai Hong 9 and Bai Hong 3 came from the same original but were divided into 6 groups, indicating that morphological traits had rich genetic diversity. As a result, comprehensive morphological status and molecular marker technology can better excavate germplasm resource base and shorten breeding process (Chen et al., 2020). In this study, we constructed a $0 / 1$ fingerprint of the alleles detected in 46 azuki bean resources, by using the primer combination method to construct the fingerprint of bean DNA. In the future research process, combining phenotype with genotype analysis will make germplasm resource identification and new variety cultivation more accurate and efficient which continuously improve the identity information of azuki bean resources and provide reliable theoretical basis and reference data for variety protection.

\section{CONCLUSION}

The association of the 9 pairs core primer selected by SSR technology can effectively distinguish 46 experimental materials. Therefore, the experimental materials can be divided into different categories according to the cluster analysis. Through $\mathrm{K}$ clustering analysis, the varieties with similar origin are often divided into the same category, providing the method for the identification of the relationship between red beans and mung beans.

\section{Funding}

This work was supported by the Department of Science and Technology of Jilin Province (No. 202002010227JC and 20210202006NC).

\section{REFERENCES}

Bai, P. (2014). Genetic diversity and association analysis of agronomic traits with SSR markers in Chinese adzuki bean. (Master thesis, Chinese Academy of Agricultural Sciences).

Chen, H., Hu, L., Yang, Y., Hao, X., Li, S., Wang, S., Wang, L., Cheng, X. (2020). Evaluation and genetic diversity analysis of agronomic traits and bruchid resistance using 481 worldwide mungbean germplasms. Journal of Plant Genetic Resources. 3: 549-559. DOI: 10.13430/j.cnki. jpgr.20190717003.

Gresshoff, P.M. (1991). Fast and sensitive silver staining of DNA in polyacrylamide gels. Analytical Biochemistry. 196(1): 8083. DOI: 10.1016/0003-2697(91)90120-I.

Hou, D., Yousaf, L., Xue, Y., Hu, J., Wu, J., Hu, X., Feng, N., Shen, Q. (2019). Mung bean (Vigna radiata L.): Bioactive polyphenols, polysaccharides, peptides and health benefits. Nutrients. 11(6): 1238. DOI: 10.3390/nu11061238.

Jacob, P., Avni, A., Bendahmane, A. (2017). Translational research: exploring and creating genetic diversity. Science Direct. DOI: $10.1016 / j$.tplants.2017.10.002.

Kita-Tomihara, T., Sato, S., Yamasaki, S., Ueno, Y., Kimura, G., Ketema, R.M., Kawahara, T., Kurasaki, M., Saito, T. (2019). Polyphenol-enriched azuki bean (Vigna angularis) extract reduces the oxidative stress and prevents DNA oxidation in the hearts of streptozotocin-induced early diabetic rats. Int J. Food Sci. Nutr. 70(7): 845-855. DOI: 10.1080/ 09637486.2019.1576598.

Kim, C., Kim, M.B., Hwang, J.K. (2020). Red bean extract inhibits immobilization-induced muscle atrophy in C57BL/6N mice. J. Med Food. 23(1): 29-36. DOI: 10.1089/jmf. 2019.4426

Luo, W., Zhang, L., Yang, K., Li, Y., Zhao, B., Li, M. and Wan, P. (2013). Construction of genetic linkage map using SSR molecular markers in azuki bean (Vigna angularis Ohwi and Ohashi). ChinaAgricultural Science. 46(17): 3534-3544.

Meng, L. (2008). Genetic parameters study of seed color and hundred-seed weight and SSR markers analysis new varieties or lines in adzuki bean (Vigna angularis) germplasm (Master thesis, Xinjiang Agricultural University).

Nordborg, M. (2000). Linkage disequilibrium, gene trees and selfing: An ancestral recombination graph with partial selffertilization. Genetics. 154(2): 923. DOI: 10.1017/S001 6672399004061.

Qiao, L., Chen, H., Wang, L., Wang, S., Cheng, X., Zhang, Y. (2015). Genetic diversity of foreign mungbean germplasm resources by agronomic characters. Journal of Plant Genetic Resources. 5: 986-993.

Qiao, L., Zheng, H., Qu, Y., Zhang, H., Yao, Y., Zhang, Y., Chen, H., Cheng, X. (2020). Genetic diversity of foreign mungbean germplasm resources by SSR markers. Molecular Plant Breeding. 22: 7577-7587.

Qu, F., Fu, J., Jin, D. and Wang, B. (1998). The molecular detection of genetic diversity. Biodiversity. 2: 64-71. 
Sato, S., Kataoka, S., Kimura, A., Mukai, Y. (2016). Azuki bean (Vigna angularis) extract reduces oxidative stress and stimulates autophagy in the kidneys of streptozotocininduced early diabetic rats. Can. J. Physiol. Pharmacol. 94(12): 1298-1303. DOI: 10.1139/cjpp-2015-0540.

Saikia, B., Kalita, P. and Das, R. (2021). Effect of simulated waterlogging condition imposed at early vegetative growth on final yield in greengram (Vigna radiata). Legume Research. 44(10): 1226-1232. DOI: 10.18805/LR-4238.

Saleem, S., Yasin, G., Haq, I.U., Altaf, A., Hussain, K. and Nawaz, K. (2021). Indole Acetic Acid (IAA) Mediated amelioration of lead $(\mathrm{Pb})$ stress-physiological indices of mung bean [Vigna radiata (L.) Wilczek]. Legume Research. 44(10): 1152-1158. DOI: 10.18805/LR-630.

Tantasawat, P.A., Poolsawat, O., Kativat, C., Arsakit, K., Papan, P., Chueakhunthod, W. and Pookhamsak, P. (2021). Association of ISSR and ISSR-RGA markers with powdery mildew resistance gene in Mungbean. Legume Research. 44(10): 1164-1171. DOI: 10.18805/LR-606.

Wang, D., Liu, J., Lu, X. and Yao, D. (2017). Comparison of microsatellite PCR gel electrophoresis staining methods. Molecular Plant Breeding. 8: 3179-3182.

Wang, L., Cheng, X., Wang, S., Liang, H., Zhao, D. and Xu, N. (2009). Genetic diversity of adzuki bean germplasm resources revealed by SSR markers. Crop Journal. 10: 1858-1864. DOI: 10.3864/j.issn.0578-1752.2009.08.004.

Wang, S., Hu, J., Cao Y., Redden, Z.X., Wang Xiaoming, L. and Amirul, I. (2001). The comprehensive evaluation and preliminary study on genetic diversity of partial Chinese adzuki bean germplasm. Journal of Plant Genetic Resources. 1: 6-11. DOI: 10.3969/j.issn.1672-1810.2001.01.002.
Wang, X.W., Kaga, A., Tomooka, N. and Vaughan, D.A. (2004). The development of ssr markers by a new method in plants and their application to gene flow studies in azuki bean [Vigna angularis (willd.) ohwi and ohashi]. Theoretical and Applied Genetics. 109(2): 352-360. DOI: 10.1007/s00122-004-1634-8.

Wu, Z., Wang, P., Pan, D., Zeng, X., Guo, Y., Zhao, G. (2021). Effect of adzuki bean sprout fermented milk enriched in $\gamma$-aminobutyric acid on mild depression in a mouse model. J. Dairy Sci. 104(1): 78-91. DOI: 10.3168/jds. 2020-19154.

Xu, H.X., Jing, T., Tomooka, N., Kaga, A., Isemura, T., Vaughan, D.A. (2008). Genetic diversity of the azuki bean [Vigna angularis (Willd.) Ohwi and Ohashi] gene pool as assessed by SSR markers. Genome. 51(9): 728-738. DOI: $10.1139 / G 08-058$.

Ye, W., Chen, S., Yang, Y., Zhang, L., Tian, D., Zhang, L. and Zhou, B. (2019). Development of SSR markers and genetic diversity analysis in mung bean. Crop Journal. 8: 1176-1188.

Zeng, C. (2010). Genetic diversity analysis on germplasms and DNA fingerprints construction in azuki bean (Vigna angularis). (Doctoral dissertation, Gansu Agricultural University).

Zhao, B., Ye, J., Jin, W., Zeng, C., Wu, B., Pu, S., Pan, J. and Wan, P. (2011). Analysis on genetic diversity and trait association of different types of azuki bean (Vigna angularis) by SSR markers. China Agricultural Science. 44(4): 673-682. DOI: 10.3864/j.issn.0578-1752.2011.04.003.

Zhao, Y., Wang, Y., Zhang, D., Wang, L. and Zuo, Z. (2017). Genetic Diversity analysis and DNA fingerprint construction based on fluorescent labeled SSR markers for mungbean varieties (Vigna radiate L.) from Inner Mongolia. Food Science. 38(16): 43-50. 